\title{
Editorial
}

\section{A new paradigm of chemotherapy for gastric cancer: speeding up, and more clinical trials to catch up}

\author{
JUNICHI SAKAMOTO \\ Department of Epidemiological and Clinical Research Information Management, Kyoto University, Graduate School of Medicine, \\ Konoe-cho, Yoshida, Sakyo-ku, Kyoto 606-8501, Japan
}

Standard chemotherapy for the treatment of gastric cancer remains controversial. Recently, in the United States, adjuvant chemoradiotherapy was shown to have a significant effect in gastric cancer patients who underwent resection [1], and the regimen has been recommended as a possible new standard treatment. In Japan, although several clinical trials comparing oral fluoropyrimidine with surgery alone as a control have been performed [2,3], definite evidence of an effective adjuvant therapeutic regimen has not yet been determined. According to the current guidelines of the Japanese Gastric Cancer Association, the only recommended standard treatment for resectable gastric cancer is surgery [4]. Even for metastatic and/or recurrent advanced gastric cancer, definite standard treatment has not been established in Western countries, nor has it been established here in Japan. In Europe and in the United States, several combination therapies containing etoposide, 5-fluorouracil (FU), leucovorin, and other drugs have shown some encouraging results $[5,6]$ for advanced gastric cancers. In Japan, a Japan Clinical Oncology Group (JCOG) study comparing three effective regimens, i.e., continuous 5-FU, irinotecan/cisplatin, and tegafur-gimestat-otastat pottassium (TS-1) is currently ongoing. To date, therefore, the key agent in mainstream chemotherapy for gastric cancer has mostly consisted of oral or parenteral fluoropyrimidine-based treatments.

Should we be content with the current status of the treatment of gastric cancer? In other major solid tumors, such as lung, breast, or colon cancer, at least two or three combination chemotherapies have been established as standard therapy, both in the adjuvant setting and for the treatment of advanced disease. For gastric cancer, there is an urgent need to expand the armamen-

Offprint requests to: J. Sakamoto

Received: March 4, 2002 / Accepted: March 20, 2002 tarium of useful agents and to accelerate the process of evaluating new treatment modalities. But what type of novel treatment modalities should we examine? And how can we make up for the delay in establishing gastric cancer treatment modalities, compared with those for other major solid tumors, without going by a roundabout way that was not successful a decade ago [7]?

Paclitaxel, which has been heralded as a unique cytotoxic agent, could be one of the most promising novel chemotherapeutic agents for gastric cancer. To understand the effect of paclitaxel, a review of its basic and clinical pharmacology is necessary. Paclitaxel is a novel diterpenoid that contains a complex ring system, called a taxane ring, coupled with a complex ester chain. This taxane derivative is a member of the tubulin-binding agent class. But, unlike other agents in the tubulinbinding family that promote microtubule disassembly, paclitaxel shifts the equilibrium toward microtubule assembly, and stabilizes microtubules by preventing depolymerization [8,9]. Experimental studies have shown that paclitaxel has multiple biological activities, and different mechanisms of action are speculated depending on the concentration of paclitaxel. At a higher dose, paclitaxel blocks dividing cells in the $\mathrm{G}_{2} / \mathrm{M}$ phase, inhibits microtubular depolymerization, and directly kills tumor cells. At a lower concentration, paclitaxel induces apoptosis [10,11] anti-angiogenesis, and immunomodulation. Paclitaxel has already been used extensively in clinical practice, as one of the key drugs for the treatment of ovarian cancer, breast cancer, and lung cancer. As paclitaxel possesses a totally different mechanism of action compared with that of the fluoropyrimidines, the drug may bring about considerable improvements in the treatment of gastric cancer.

The safety profile of paclitaxel has been well established. The incidence of severe hypersensitivity reactions observed in the initial clinical trials has been significantly reduced by the prophylactic administration of antiallergy medication, including a corticosteroid 
coupled with an $\mathrm{H}_{1}$ and an $\mathrm{H}_{2}$ histamine antagonist [12]. Neutropenia, the most frequent side effect of paclitaxel, has been shown to be related to the duration of serum concentration over $50 \mathrm{nM}$, and not to be related to the peak concentration or AUC [13]. That is why a longer, 24-h, infusion of paclitaxel produces more myelosuppression than a shorter, 3-h infusion [14]. The occurrence of peripheral neuropathy, on the other hand, has been shown to be dependent on peak concentration and on the cumulative dose administered.

Recently, several studies of paclitaxel administered as a 3-h infusion in patients with gastric cancer have been reported. In an early phase II study in 15 patients with refractory gastric cancer, a $20 \%$ response rate was observed [15]. In a subsequent late phase II study, a response was observed in 14 of 60 patients. Of note, in this study, $27 \%$ of patients who had received prior chemotherapies (including either a fluoropyrimidine, cisplatin, or both agents) showed responses to paclitaxel [16]. In the second late phase II study, published in this issue of Gastric Cancer [17] a 28\% response was reported in 32 patients with advanced gastric cancer. It should also be noted that, in this study, a $24 \%$ response rate was obtained in patients who had previously received chemotherapy. Based on these results, there seems to be a lack of cross-resistance between fluoropyrimidines and/or cisplatin with paclitaxel in patients with advanced gastric cancer.

The main toxicities reported during these late phase II studies of paclitaxel in patients with advanced gastric cancer were grade 3/4 neutropenia and leucocytopenia. These toxicities are considered to be manageable with the proper use of granalocyte-colony-stimulating factor (G-CSF). No significant non-hematologic toxicity has been observed in these late phase II studies.

Can we identify patients prospectively who would respond better to paclitaxel therapy? A preliminary study has reported that tumors in patients who show microtubule DNA mutation are resistant to paclitaxel, and a randomized clinical trial to evaluate the genetically segregated effect of paclitaxel has been started in Europe [18].

The phase II clinical trial for patients with gastric cancer published in this issue of Gastric Cancer [17] is of interest for three reasons. First, paclitaxel, a drug that has a totally different mechanism of action compared with these of other gastric cancer chemotherapeutic agents, was confirmed to be effective. Second, investigators have reported the rapid transition of intravenous paclitaxel to the peritoneal cavity [19]. This effect could, potentially, be of importance in eliminating refractory peritoneal metastasis, which could be fatal in this disease. Finally, enrollment was completed after the registration of 32 patients, instead of the 60 patients required in the preliminary design. The application of Bayes' theorem [20], using Jeffreys' prior distribution method [21], enabled the definitive confirmation of the effect of paclitaxel with a smaller sample size than that estimated in the initial planning of the trial.

Should paclitaxel be used routinely for the treatment of gastric cancer? Some clinicians and investigators will promote a more extensive use of paclitaxel for therapy of advanced gastric cancer outside clinical trials, because it has been shown that a substantial proportion of refractory tumors regress following the administration of the drug as a 3-h infusion. However, it is my contention that, at this time, paclitaxel should be used mainly in clinical trials. As we have learned from our previous experience in gastric cancer, rapidly adopting a new treatment could produce stagnation and confusion rather than progress. The adoption of a new standard chemotherapy regimen in patients with gastric cancer should be based on the results of extensive clinical trials.

Which additional clinical trial(s) should be performed? In this author's opinion, three different avenues should be explored and run in parallel. First, the most appropriate schedule for paclitaxel administration should be investigated. Recently, the weekly administration of paclitaxel, compared with tri-weekly administration, was reported to achieve higher dose intensity with reduced toxicity [14]. Second, clinical trials should investigate the best therapeutic sequence for preexisting regimens and paclitaxel, as has been done by the GRECOR group for metastatic colorectal cancer [22]. It might be of interest to determine the response rate and time to progression in patients with gastric cancer who are receiving paclitaxel or pre-existing fluoropyrimidines or cisplatin as first-line therapy. Third, the most important approach should be to investigate combinations of paclitaxel with other chemotherapeutic drugs used in gastric cancer. As discussed above, paclitaxel has been used extensively in combination therapies for the treatment of other solid tumors. Therefore, the same approach should be undertaken in patients with gastric cancer. Any attempts to determine the safety and efficacy of combination therapy that includes paclitaxel with other cytotoxic or cytostatic agents for advanced and even for resected gastric cancer must be strongly encouraged and hastened. Although Health Authorities may require the completion of single-agent paclitaxel comparative trials and recommend the initiation of new investigations only after the results of those trials are obtained, the author believes that such a process, although scientifically appropriate, is too slow to allow us to catch up with the current progress of basic and clinical science.

Therefore, we should not hesitate to speed up the process and start more clinical trials to investigate dosing, scheduling, alternative sequences, and combined treatments with paclitaxel, which has a totally 
different mechanism of action from these of already approved agents for gastric cancer. Too much prudence might sometimes be disturbing for gastric cancer research, as well as for the patients who could benefit from its findings.

\section{References}

1. Macdonald JS, Smalley SR, Benedetti J, Hundahl SA, Estes NC, Stemmermann GN, et al. Chemoradiotherapy after surgery compared with surgery alone for adenocarcinoma of the stomach or gastroesophageal junction. N Engl J Med 2001;10:725-30.

2. Nakajima $T$, Nashimoto A, Kitamura M, Kito T, Iwanaga T, Okabayashi K, et al. Adjuvant mitomycin and fluorouracil followed by oral uracil plus tegafur in serosa-negative gastric cancer: a randomised trial. Lancet 1999;354:273-7.

3. Nashimoto A, Nakajima $T$, Furukawa $H$, Kitamura $M$, Yamamura Y, Kinoshita T, et al. Randomized trial of adjuvant chemotherapy with MCF followed by oral fluorouracil in serosanegative gastric cancer: JCOG Study 9206-1. Proc ASCO 2001;20: 164a. (abstract 653).

4. Japanese Gastric cancer Association. Introduction to JGCA gastric cancer treatment guidelines. Tokyo: Kanehara; 2001. pp. 46.

5. Webb A, Cunningham D, Scarffe JH, Harper P, Norman A, Joffe $\mathrm{JK}$, et al. Randomized trial comparing epirubicin, cisplatin, and fluorouracil versus fluorouracil, doxorubicin, and methotrexate in advanced esophagogastric cancer. J Clin Oncol 1997;15:261-7.

6. Vanhoefer U, Rougier P, Wilke H, Ducreux MP, Lacave AJ, Cutsem EV, et al. Final results of randomized phase III trial of sequential high-dose methotrexate, fluorouracil, and doxorubicin versus etoposide, leucovorin, and fluorouracil versus infusional fluorouracil and cisplatin in advanced gastric cancer: a trial of the European Organization for Research and Treatment of Cancer. Gastrointestinal Tract Cancer Cooperative Group. J Clin Oncol 2000;18:2648-57.

7. Sakako M, Maruyama K. Adjuvant chemotherapy of gastric cancer has to be fundamentally reconsidered (in Japanese). Igaku-no-ayumi (J Clin Exp Med) 1990;153:664-8.

8. Shiff PB, Horwits SB. Taxol stabilizes microtubules in mouse fibroblast cells. Proc Natl Acad Sci USA 1980;77:1561-5.
9. Rowinsky EK, Cazenave LA, Donehower RC. Taxol: a novel investigational antimicrotubule agent. J Natl Cancer Inst 1990;82: $1245-59$.

10. Ringel I, Horwitz SB. Paclitaxel affects microtubule dynamics and apoptosis. Adv Oncol 1999;15:11-17.

11. Torres K, Horwitz SB. Mechanisms of taxol-induced cell death are concentration dependent. Cancer Res 1998;58:3620-6.

12. Grem JL, Tutsch DT, Simon KJ, Alberti DB, Willson JK, Tormay DC, et al. Phase I study of taxol administrated as a short IV infusion daily for 5 days. Cancer Treat Rep 1987;71:1179-84.

13. Gianni L, Kearns CM, Giani A, Capri G, Vigano L, Locatelli A, et al. Nonlinear pharmacokinetics and metabolism of paclitaxel and its pharmacokinetic/pharmacodynamic relationship in humans. J Clin Oncol 1995;13:180-90.

14. Glantz MJ, Choy H, Akerley W, Kearns CM, Egorin KM, Rhodes $\mathrm{CH}$, et al. Weekly paclitaxel with and without concurrent radiation therapy: toxicity, pharmacokinetics, and response. Semin Oncol 1996;23:128-35.

15. Ohtsu A, Boku N, Tamura F, Muro K, Shimada Y, Saigenji K, et al. An early phase II study of a 3-h infusion of paclitaxel for advanced gastric cancer. Am J Clin Oncol 1998;21:416-19.

16. Yamada Y, Shirao K, Ohtsu A, Boku N, Hyodo I, Saitoh H, et al. Phase II trial of paclitaxel by 3-h infusion for advanced gastric cancer with short premedication for prophylaxis against paclitaxel-associated hypersensitivity reactions. Ann Oncol 2001; 12:1133-7.

17. Yamaguchi K, Tada M, Horikoshi N, Otani T, Takiuchi H, Saitoh S, et al. Phase II study of paclitaxel with 3-h infusion in patients with advanced gastric cancer. Gastric Cancer 2002;5:90-5.

18. Monzo M, Rosell R, Sanchez JJ, Lee JS, O'Brate A, GonzalezLarriba JL, et al. Paclitaxel resistance in non-small-cell lung cancer associated with beta-tubulin gene mutations. J Clin Oncol 1999;6:1786-93.

19. O'Boyle KP, Wang Y, Schwartz YW, Regl DL, Einzig A, Dutcher JP, et al. Development of two radioimmunoassays to detect paclitaxel in sera and in cerebrospinal, ascitic, and pleural fluids. Cancer 1997;79:1022-30.

20. Savage LJ. The foundation of statistics. New York: Wiley; 1954.

21. Jeffreys H. Theory of probability. 3rd. ed. Berkeley; University of California Press, 1961.

22. Tournigand C, Louvet C, Andre T, Achille E, Lledo G, Flesch M, et al. FOLFIRI followed by FOLFOX followed by FOLFIRI in metastatic colorectal cancer: which is the best sequence? safety and preliminary efficacy results of a randomized phase III study. Proc ASCO 2000;19:245a (abstract 949). 